\title{
New Hypotheses and Viewpoints for Atherosclerosis in 2013 Faruk Erzengin*
}

Professor of Internal Medicine and Cardiology, Previous Dean of Medical Faculty of Istanbul, University of Istanbul, Departmet of Cardiology, Çapa, Istanbul, Turkey

\begin{abstract}
Formation of atherosclerotic and calcified plaque begins and grows up not only beneath the endothelium (intimae), but also in the adventitial subepithelium on the coronary arteries.
\end{abstract}

A new innovative combined drug (polypill) is certainly releases, regress and prevents of formation of the atherosclerotic and calcified plaque of arteries.

Keywords: Atherosclerocalcification; Atherosclerocalcific plaques; Multi slice computed tomography; Coronary atherosclerosis; Erzengin's polypill

\section{Introduction}

Despite steady progress in the treatment of atherosclerotic and atherothrombotic cardiovascular disease, people are still dying of coronary artery diseases and myocardial infarctions. These two problems remain the leading causes of morbidity and mortality particularly in the industrialized countries. Atherosclerosis is the descriptive term for thickened and hardened lesions which have lipids and calcifications of the intimae and media of elastic and muscular arteries. It has to be pointed out that the primary cause of arterial atherosclerocalcification is still unknown as yet. At the end, it has been accepted that, atherosclerotic and calcified lesions start, occur and grow up within the innermost layer of the arteries (at the intimae). It has been firstly accepted in 1995 by American Heart Association (AHA) that, the earliest lesions of atherosclerosis (fatty streaks or type III lesions) were present in the intimae of aorta from childhood. Today, we know that atherosclerosis begins as early as foetal life especially in foetuses of hypercholesterolemic mothers [1]. In any arterial bed of intimae, formation and progression of the atherosclerotic plaques and calcifications were well documented by many authors; like V. Fuster, E. Falk [1-4]. and were sub divided several phases by them which are summarised such as at below. Most lipids deposited in the atherosclerotic lesions (atherosclerotic components) are derived from plasma low-density lipoproteins (LDLs) that enter the vessel wall through the injured or dysfunctional endothelium. In normal population, from 5 to 10 years of ages, fatty streaks often are present in the aorta and coronary arteries, they were accepted the initial points of plaque development. Type I lesion consist of macrophage-derived foam cells that contain lipid droplets; type II lesions contain macrophages and smooth muscle cells with extra cellular lipid deposits; and type III lesions contain smooth muscle cells surrounded by extracellular connective tissue, fibrils, and lipid deposits; Type IV plaques consist of confluent cellular lesions with a great deal of extracellular lipid intermixed with fibrous tissue, whereas Type Va plaques possess and extra cellular lipid core covered by a thin fibrous cap. Either phase III or IV plaque can evolve into fibrotic plaques of phase $\mathrm{V}$, characterized by type $\mathrm{Vb}$ or $\mathrm{Vc}$ lesions, with or without predominant calcification. Type VI occlusive thrombus overlying a superficial erosion of a markedly stenotic and fibrocalcific plaques of phase V [2-6]. In Phase II-V vulnerable lipid-rich plaques are prone to disruption because of the fact that their high lipid content. Raised plaques appear later, by 20 years of age are present in areas, such as, on the endothelium of proximal left anterior descending (LAD) coronary artery, where fatty streaks are most prevalent in early life [3]. The surface of intimae opened human all arteries are approximately 800 square meters, produce more than 250 active substances and at the end reveals the presence of several types of plaque. Some are flat yellow dots or lines (fatty streaks), and the others are raised above the surface as oval humps, which range in colour from white to yellow (raised fibro lipid plaques) [1-6]. An early atherosclerotic lesion (fatty streak or type III lesion) is the aortic root and coronary arteries, the lesion consists of lipid-laden monocytederived macrophage foam cells and a few $\mathrm{T}$ lymphocytes beneath an intact endothelium. It has been accepted that, only endothelial cells, monocyte-derived macrophages or foam cells and a few T cells participate in the early inflammatory and immune response, giving rise to early atherosclerotic lesions. This early atherosclerotic lesions (fatty streak or type III lesion) represent a dynamic balance of the entry and exit of lipoprotein entry by endothelium injury resulting in a predominance of lipoprotein exit and final scarring [1-6]. In disease progression, the oxidative modified LDL (oxLDL), the inflammatory and immune response is accompanied by a fibro proliferative response in which the vascular smooth muscle cells play dominant roles [111]. OxLDL has many proinflammatory properties which explain the local up regulation of inducible endothelial cell adhesion molecules, even before lesion formation, in hypercholesterolemic atherosclerosis and atherothrombotic events [1-6]. OxLDL has proinflammatory and cytotoxic effects. It was recognised by the macrophage scavenger receptor promoting intracellular lipid accumulation and foam cell formation. Such as the endothelial dysfunction or/activation (vascular cell adhesion molecule 1), monocyte adherence, injuring lipoprotein retention, oxLDL, İnflammatory/immune response, macrophage scavenger foam cell formation, endothelial shear stress, turbulent flow, high blood pressure on the vessels' walls and the role of nitric oxide (NO), prostacyclin, endotheline-1, acetylcholine, vascular cell adhesion molecule-1 (CAM-1), cell adhesive molecules (CAM or surface glycoproteins), intercellular adhesion molecule-1(ICAM-1) promote or enter though the injured or dysfunctional endothelium and sub endothelial lipid accumulation and et the end lipid core formations occure. As soon as monocytes (macrophages) adhere to the surface of endothelium (intimae) with the specific molecules, such as monocyte chemotactic protein-1(MCP-1) and macrophage colony-stimulating

${ }^{*}$ Corresponding author: Faruk Erzengin, Departmet of Cardiology, Previous Dean, University of Istanbul, Istanbul Medical Faculty, Istanbul, Turkey, Tel: +90 53245351 79; E-mail: farukerzengin@gmail.com

Received November 19, 2013; Accepted December 30, 2013; Published January 25, 2014

Citation: Erzengin F (2014) New Hypotheses and Viewpoints for Atherosclerosis in 2013. General Med 2: 130. doi: 10.4172/2327-5146.1000130

Copyright: (c) 2014 Erzengin F. This is an open-access article distributed under the terms of the Creative Commons Attribution License, which permits unrestricted use, distribution, and reproduction in any medium, provided the original author and source are credited. 
factor (M-CSF) [1]. Macrophage or foam cells, before or after their death, can liberate and accumulate many products including oxLDL and free radicals. Chronic minimal endothelial injury or dysfunction, plasma low-density proteins enter through the injured endothelium, leading to accumulation of lipids (oxLDL) and macrophages are produce atheroma mass or atheromatous component by depend upon of heredity, diabetes, hypercholesterolemia, in tobacco smoke, hypertension, abdominal obesity, gender, aging, high plasma uric acid level, chemical irritants, infection, high level svCRP and fibrinogen, circulating vasoactive amines, immune complexes, etc.

So this classical pathway or famous well known cascade of atherosclerosis brings about the formation of the atheroma in 1995 by AHA of atherosclerotic lesions [1-3]. This pathway of formation of atherosclerosis and calcifications almost universally were accepted in the intimae (under the endothelium). In spite of the fact that, there is no any doubt of this classical pathway or rule of the cascade of formation of atherosclerocalcifications. Atherosclerosis is a focal disease in the intimae of large and medium sized systemic arteries. It has been well documented that, focal calcification in atherosclerotic plaques is very common in humans' arteries and nicely shown that coronary calcification in adults is almost always atherosclerosis related intimae until today [8]. We have recently shown that by the Multi Slice Computed Tomography (MSCT-320) using within a device which has a computerised magnifying glass. On the other hand making a comparison between MSCT, coronary angiography, perioperative findings and histo-pathological data which detected by light and electron microscopy and environmental SEM (By using microtom, slaces samples might be taken, across the walls of samples clasical histo-pathological routine investigation and addition to chemical analysis can be taken by means of energy dispersive X-ray mobile monitoring the surface of speciment by SEM). We strongly emphasized that, the classical cascade of the atherosclerotic and calcified plaques formation begin and grow up not only beneath the intimae (sub endothelium) or media but also in adventitial epithelium on the coronary arteries. We have surprisingly determined that the adventitial side of the calcification develops much faster and severer than that of the intimae and medial side of on the arterial vessels' walls.

MSCT of coronary angiography with 64 slice technology, first described by Leschka et al. [7]. Currently, MSCT 320 with magnifying glass is a very important tool for the non-invasive evaluation of coronary arterial pathologies. Moreover, the MSCT is an excellent and important technique to show that the place and size of the formation of plaques and calcifications on the arteries [7-8]. It has been well documented that in the last three decades, the cascade of atherosclerotic plaques formation start from beneath the endothelial cells of coronary arteries [1-25]. The media calcification of the coronary arteries was also described as Mönckeberg's Sclerosis many years ago and it is rare in coronary arteries and frequently occurs in other arteries (particularly arteries of the leg and aorta) $[1,2,4,8,16,17]$. All calcification of arteries are directly related with atherosclerotic burden or component $[4,8,16,17]$. Until today it has not written yet anything about our findings of adventitial atherosclerosis and calcifications [18-21,24].

We have recently shown that by MSCT-320, the formation of the atherosclerotic and calcified plaques begin not only in the intimae but also under the adventitial epithets on the coronary arteries, surprisingly. The adventitial calcifications grow up the most quickly than the sub endothelial (the intimae) calcified plaques. On the other hands, the medial calcifications much frequently grow up on the aorta and its main branches, such as carotid, vertebral, cerebral, renal, mesenteric, ilio-femoral, and peripheral arteries. Interestingly, the formation of calcified plaque much frequently begins beneath the sub-epithelium of the adventitia and quickly grows up towards to the lumen of arteries and keeping same classical and accepted cascade on the coronary arteries. These calcifications mostly begin in the middle of the atheromatous component. The cholesterol (particularly oxLDL) and macrophage cells easily arrive to the adventitia vase-vasorums of the coronary arteries from blood and/or by diffusion from epicardial fat accumulation araund the heart appears in three different types: a) Intra cellular in the heart, b) Epicardial and, c) Pericardial around the adipouse tissue of the heart. Intracellular fat is the microscopic lipid accumulation within the cytoplasm cardiac myocytes and can be the result of myocardial ischemia, cell damage and/or cell death. The epicardial fat tissue is located between the outer wall of the myocardium and the visceral layer of pericardium. The Pericardial fat exists anterior to the epicardial fat layer and therefore located between visceral and parietal pericardium. Due to the close anatomic relation between myocardium and the epicardial fat, the two tissues share the same microcirculation. By vasevasorums, diffusion way or potential interactions through paracrine and vasocrine mechanism between epicardial fat and adventitia of coronary arteries or myocardium are strongly suggested [25-27].

On the other hand we have proved that; previously performed Multi Slice Computed Tomography has plaid an important role for patients as a guide for detecting size of divices and to perform elective procedure of coronary lesions during implantation of stent in the catlab.

Most of the risk factors that apply to the atherosclerotic vascular diseases in all arteries. Herein nine different and consecutive cases (Figures 3-12) have been shown which were investigated by MSCT

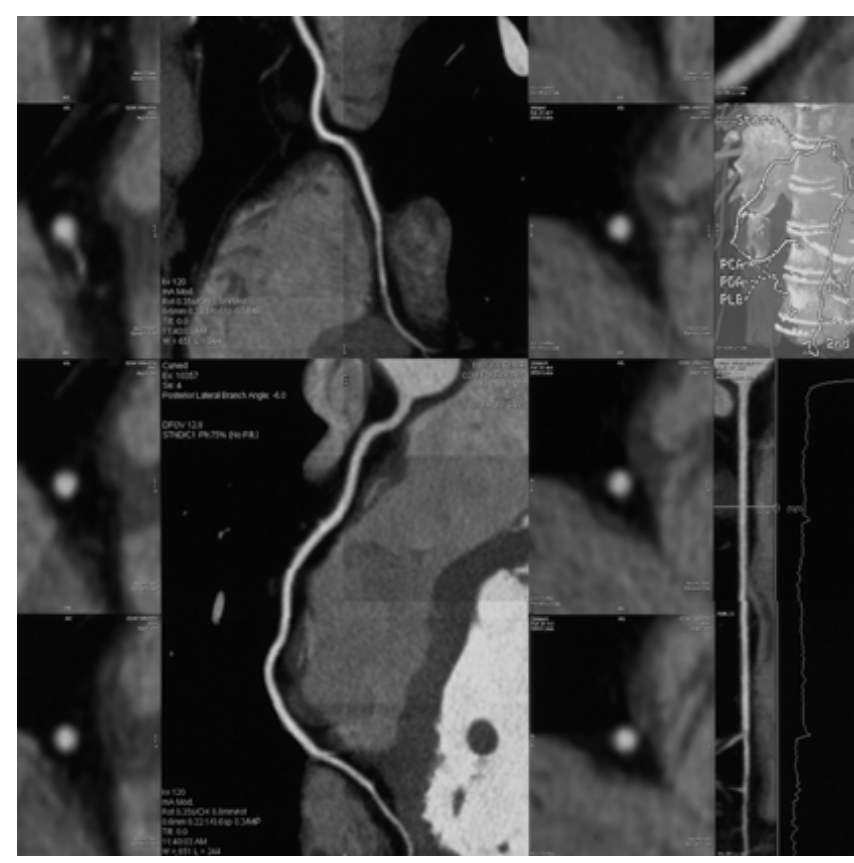

Figure 1: A normal Right Coronary Artery (white arrow) is shown by MSCT with no atherosclerotic plaque or calcification . : 


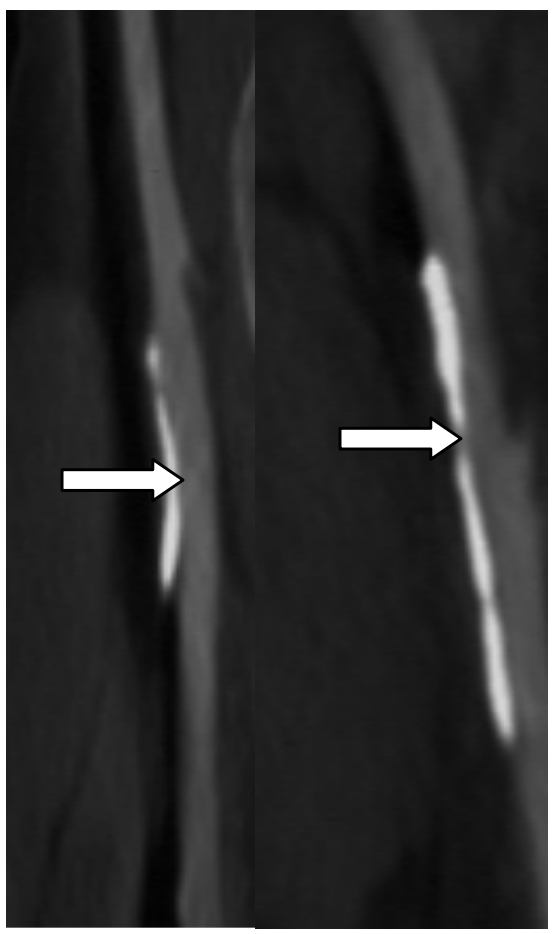

Figure 2: Adventitial atherosclerocalcifications (atheromatheous components) of on the femoral arteries of Guinea pigs. The grey areas are soft plaque (lipids core), the white areas are calcified plaques.

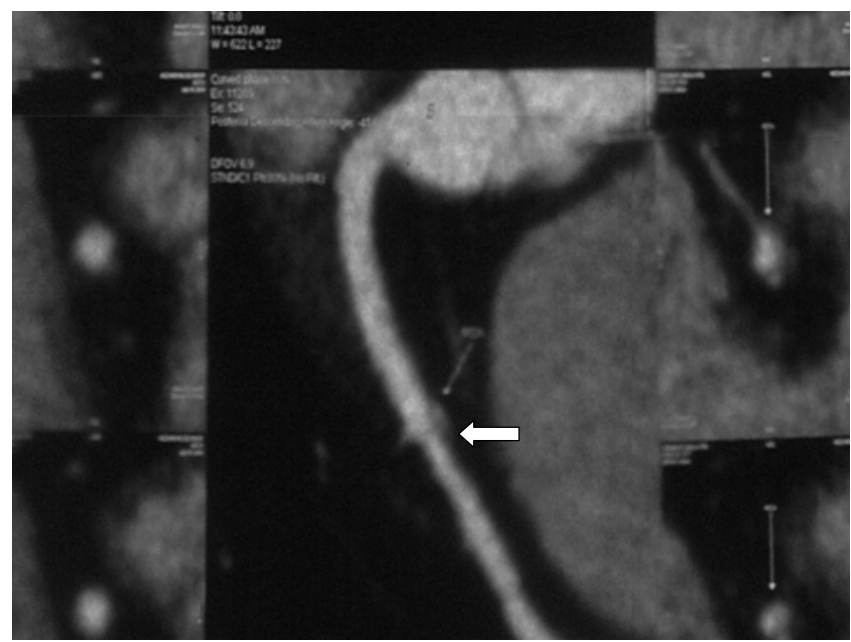

Figure 3: Adventitial soft plaque on the right coronary artery of a 48 years old man (is shown white arrow).

34-320 and conventional coronary angiography. The atherosclerotic plaques and calcifications begin and grow up beneath the adventitial epithelium through the lumen of vessels on the coronary arteries. It means, our recent studies showed in most patients (approximately \% 51 ), the calcified plaques were started just beneath of the epithelium on the adventitia and grown forward to the lumen of the arteries. Of course, they can make a severe stenosis or occlusion of arterial lumen at the end. In comparison with IVUS, Stephan Achenbach et al. found a sensitivity of $\% 82$ to detect coronary artery segments containing atherosclerotic plaque in patients without significant coronary artery stenosis [15]. MSCT might be useful for the characterization of human coronary plaque morphology by determining tissue density within the lesion non-invasively. Also MSCT is a unique non-invasive method for the detection of coronary atherosclerotic plaque morphology and for the diagnosis of silent ischemia, lumen narrowing calcification of adventitia [18]. Molecular imaging most probably will be sort it out and help us to show this complex morphology in the near feature.

So we have firstly described here that the opposite side of the atherosclerotic new location and pathway, which is, formation of the atherosclerotic plaques start not only from intimae but also from adventitial epithelium on the coronary arterial walls. The MSCT-320 is a unique technique to show that unknown opposite side of the classical cascade and pathway of the atherosclerosis. This finding is described firstly in our randomised comparative coronary studies making a comparison between the invasive and non-invasive techniques which is not described in the Literature. We have emphasized that MSCT320 with magnifying glass is an important and unique device and is an important tool for non-invasive evaluation of coronary arterial pathologies and plaque formations.

In the first patient, a normal coronary artery taken by MSCT was shown in Figure 1. In Figure 2, the adventitial atherosclerocalcifications (atheromatheous components) of on the femoral arteries of Guinea pigs have been shown. In the third cases (Figure 3), a soft plaque of atheroma on the right coronary artery of adventitia it takes place. In Figure 4, the atherosclerotic soft and vulnerable plaque (atheromatous component) starting from adventitia of the mid segmental part of the Left Anterior Descending (LAD) artery and multiple small adventitial calcifications were shown that just beneath the subepithelium of the adventitia on the (LAD). In Figure 3-12 have been shown from minimal to severe calcifications and some degree of

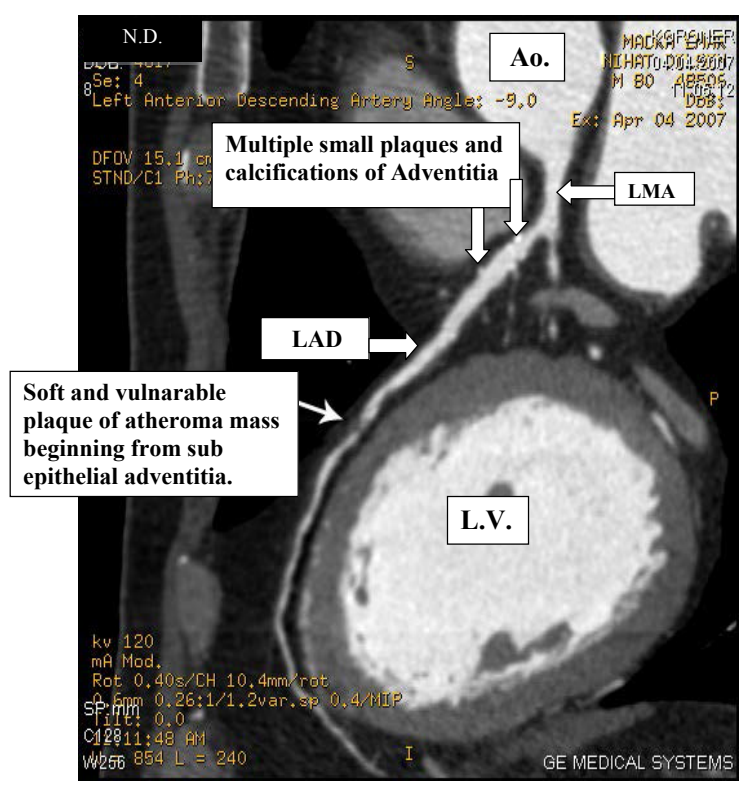

Ao: Aota, LAD: Left Anterior Descending, LMA: Left Main Artery, LV: Left Ventricle.

Figure 4: The adventitial atherosclerotic plaques with minimal calcifications on the LAD artery without stenosis and a vulnerable soft plaque of atheromatous component starting from sub epithelial area of adventitia on the mid LAD artery with significant stenosis. 


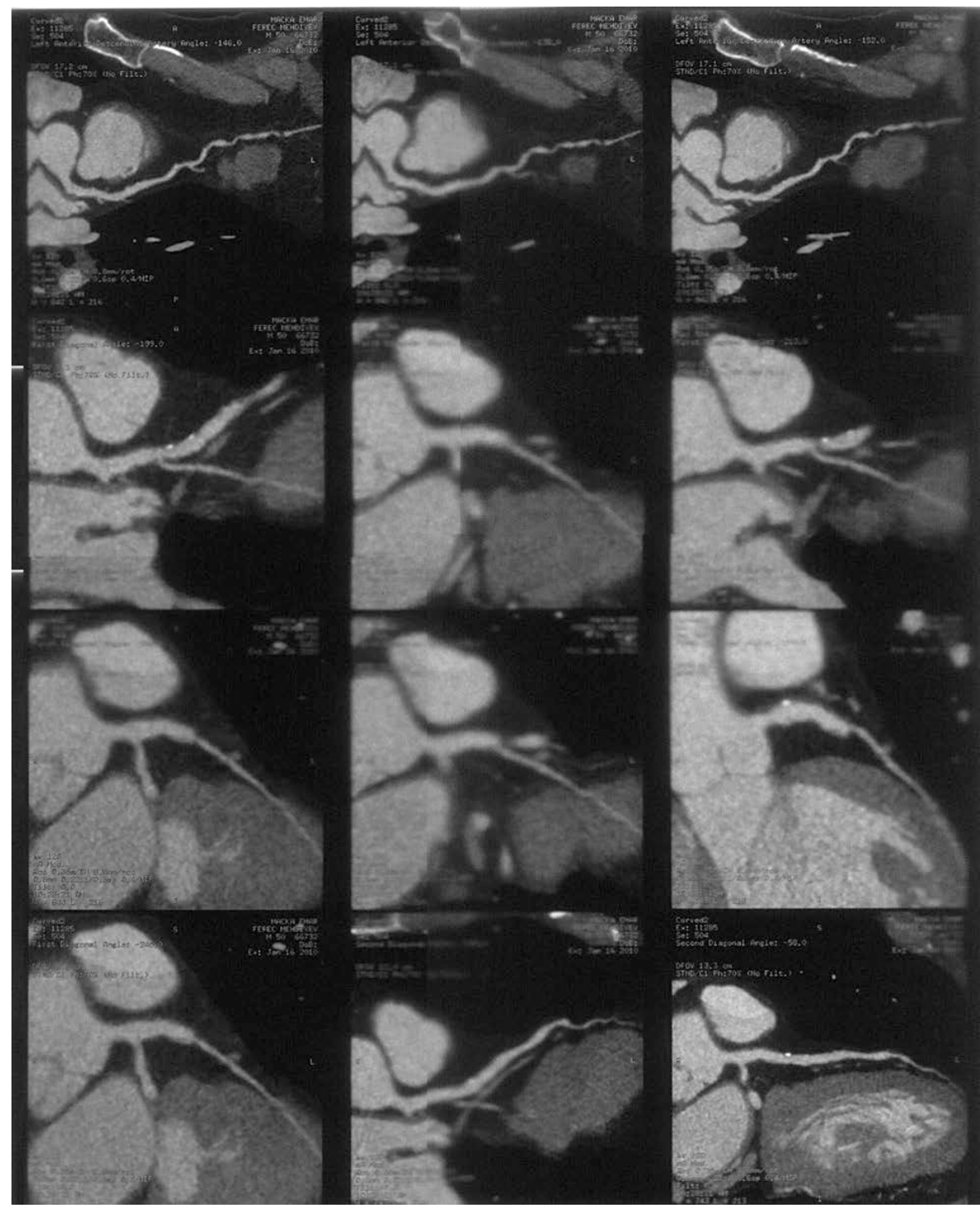

Figure 5: The adventitial small calcifications on the LAD (are shown white arrows).

atheromateous cmponent were shown which started and were taken placed just beneath the epithelium of adventitia and grown towards to the coronary arterial lumen making some degree of obstructions on the Left Main, LAD and Circumflex (Cx) of coronary. MSCT320 is very useful device for the characterization of human coronary plaque morphology by determining tissue density within the lesion non-invasively. Also MSCT is a unique non-invasive method for the diagnosis of silent ischemia and asymtomatic myocardiai infarctions dueto lumen narrowing calcification of adventitia.

In our studies totaly 917 patients were investigated by MSCT. In the first subgroop of them (224 randomised consecutive cases, $94 \mathrm{~F}$, $130 \mathrm{M}$ ), MSCT and invasive coronary angiography were performed to make a comparison between their results. At the end of the this investigation, 144 patients had stent implantations, 32 patients underwent coronary bypass sugery and 48 patients treated medically. The total groop of 917 patients, some cases underwent MSCT and invasive coronary angiography, IVUS, coronary arterial byopsy in the operating room and the taken trough cut coronary mateherial were send as soon as possible the ligt, electron and SEM microscopic investigations were performed to see localisation of atheromas, atheromatous components and calcificatins.

I gave four international lectures about the formation of atherosclerosis. One of them at the $78^{\text {th }}$ EAS Congress in HamburgGermany on June 20-23, 2010 and the second of them at the $3^{\text {rd }}$ World Medicine Congress in Nalchik, KBSU in Russia on September 20-23, 2010, the others of them were in Turkey in the International Congress of Cardiology on 2011 and 2012 [19-21,23,25]. The title was; a new, unknown and a surprise localisation of athereosclerosis (Erzengin's Adventitial Athereosclerocalcification) [20] and Erzengin's Polipill [25]. Athereosclerocalcification is one of the major causes of progressive degradation of the human arteries and target organs. It has to be pointed out that the primary cause of arterial atherosclerocalcification is still unknown as yet. It progresses insidiously and its damaging effects of human organism remain unknown for a long time before symptoms 


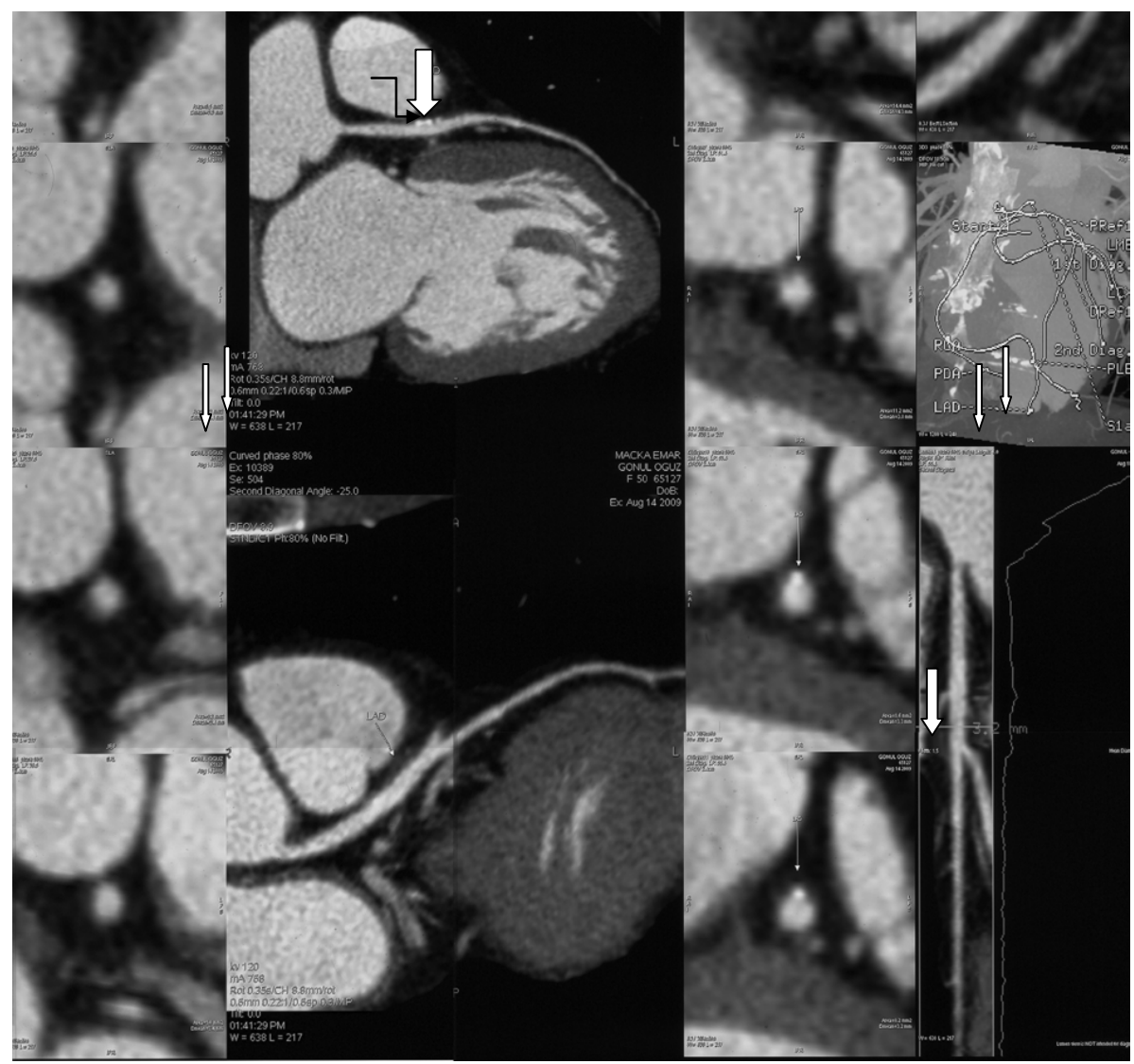

Figure 6: The atheroma mass; minimal calcifications surrounded by some degree of (lipids) soft plaques (have been shown with black and white tiny arrows) were taken place just below the adventitia of LAD artery. (In this case, LAD and Cx arteries were emerging from two separated orifices as a congenital coronary anomaly.).

of cardiac, cerebral, peripheral or other organs the ischemia develops. Ischemia creates an imbalance between oxygen supply and demand of the tissue and is usually related to atherosclerocalcificatic obstruction of the arteries with more or less severe luminal stenosis that may eventually be complicated by the formation of clots. Knowledge of its pathogenesis and of the early warning symptoms is imperative to interrupt progression of disease that has not only a high mortality, but is also the cause of several disabling diseases. It has been shown that the cell biology of the arterial wall suggest that injuries of the endothelium, induced by the concurrent action of mechanic and humoral factors, stimulate the formation of atherosclerocalcifications.

Today we know that atherogenesis is influenced by numerous factors more than 250. Atherosclerosis was defined by Osler in 1908 for the first time [8]. Osler mentioned that inflamation and infection were probably responsible for the etiology of atherosclerosis. After that, the classical endothelial pathway of the atherosclerosis was accepted as the reason of atheroma formation in 1995 by AHA. Until today, it has been accepted that the atherosclero-calcification is located in the subendothelial area (intimae). Only Mönckeberg demonstrated that the location of atherosclerosis in the media in a limited group of patients several years ago $[8,25]$. According to the classical approach; it was known that this progressive process starts and grows up from intimae, which is inside the lumen of arteries and also mostly progresses as time passes.
However, our team recently demonstrated adventitial localization of atherosclerocalcification by 64-320 Multi Slice Computed Tomography (MSCT) with a magnifying glass on 917 patients. Our conclusion was; the MSCT 320 is very useful technique for the characterization of human coronary plaques morphology because the MSCT can determine tissue density within the lesion non-invasively. Additionally, the MSCT, a unique and non-invasive method, is used for the detection of coronary atherosclerotic plaque morphology (particularly vulnerable plaque) and calcification score, also for the diagnosis of silent ischemia and asymptomatic myocardial infarctions caused by lumen narrowing calcification starting from the adventitia [18].

Thus, our surprising findings are; the formation of the atherosclerotic and calcified plaque begins and grows up not only in the intimae or media (Mönckeberg's Sclerosis), but also on the adventitia (Erzengin's Arterial Atherosclerocalcifications) of the coronary arteries and/or all of the medium and large arteries [20]. We suggest that the formation of the Adventitial Atherosclerocalcifications is caused by vase-vasorums and/or diffusion from the adipose tissues around the pericardium. white and brown adipose tissues around the heart contain more than 250 atherogenic substrates such as macrophages, oxLDL, etc.

As a result of our studies these views were generated step by step as our knowledge has evolved during the last 5 years. It is the first 


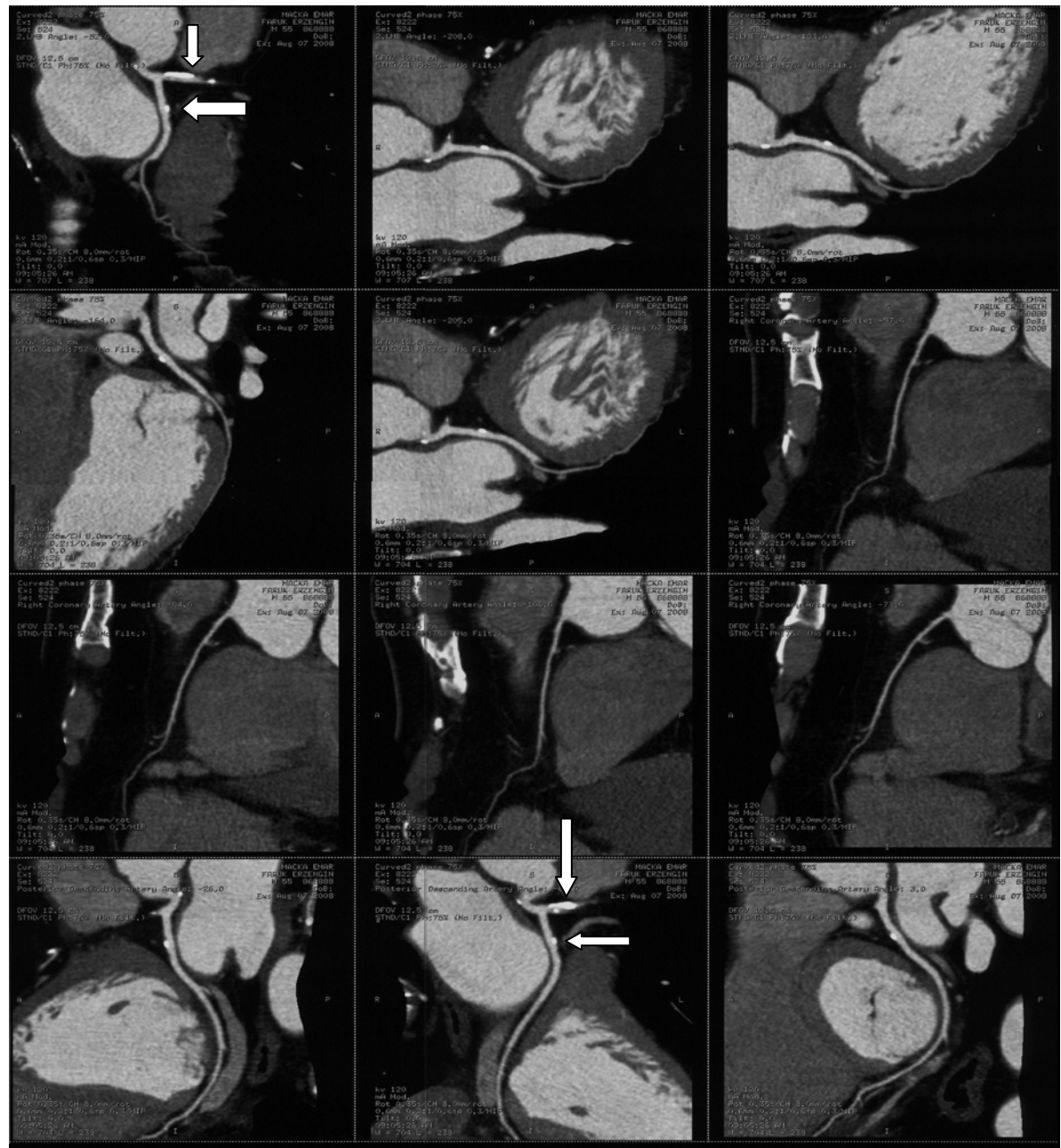

Figure 7: Middle-size calcifications on the LAD and $C x$ arteries progressing from adventitia with narrowing of the lumen has been shown (with arrows) the other case.

time in the Literature that the Adventitial Athereosclerocalcification is demonstrated by the MSCT and proved pathologically in our research laboratories. In addition to these, coronary angiography, IVUS, perioperative findings, histopatological data detected by light, electron microscopy and environmental SEM have been used for verifying the results.

It doesn't matter, the atheromatheous components' formation occure by which way or wherever on the which tunicas of the artheries, the results almost always same. Formation of the atheroma mass depends on the heredity, hypercholesterolemia, diabetes, smoking, hypertension, abdominal obesity, gender, aging, hyperurisemia, chemical irritants, infections, high svCRP and fibrinogen, circulating amines, immune complexes and etc.

Athereosclerocalcification is one of the major causes of progressive degradation of the human arteries and target organs. It progresses insidiously and its damaging effects of human organism remain unknown for along time before symptoms of cardiac, cerebral, peripheral or other ischemia develop.

For many years, atherosclerotic plaque formation has been accepted as a dynamic, progressive process and a very dangerous, killer disease which is never prevented, stabilized or treated because of its dangerous progression. Unfortunately, the atherosclerosis still has the highest morbidity and mortality rates all over the world.

According to our new clinical and laboratory findings; we believe that athereosclerotic and calcified plaques can be stabilized and totally treated with only drug therapy wherever their localisation on the arteries are. It means that, in addition to the current treatment, our polypill combination can totally cure the atherosclerosis and prevent plaque formation permanently. So, we have proved that this silent killer process is regressive, preventable and totally curable with only combined drug therapy.

Today, I am going to write about a new additional treatment (Erzengin's Polypill) of athereosclerocalcifications [25]. In here I will present our new medical combination therapy against this progressive, dynamic and killer disease. Atherosclerocalcifications cause deaths and disabilities by occluding the arteries of several vital organs. In this manner, this non invasive treatment prevents this virulent diseases which require expensive, palliative and high-risk surgical procedures and/or invasive interventions. The medical treatment would also be 


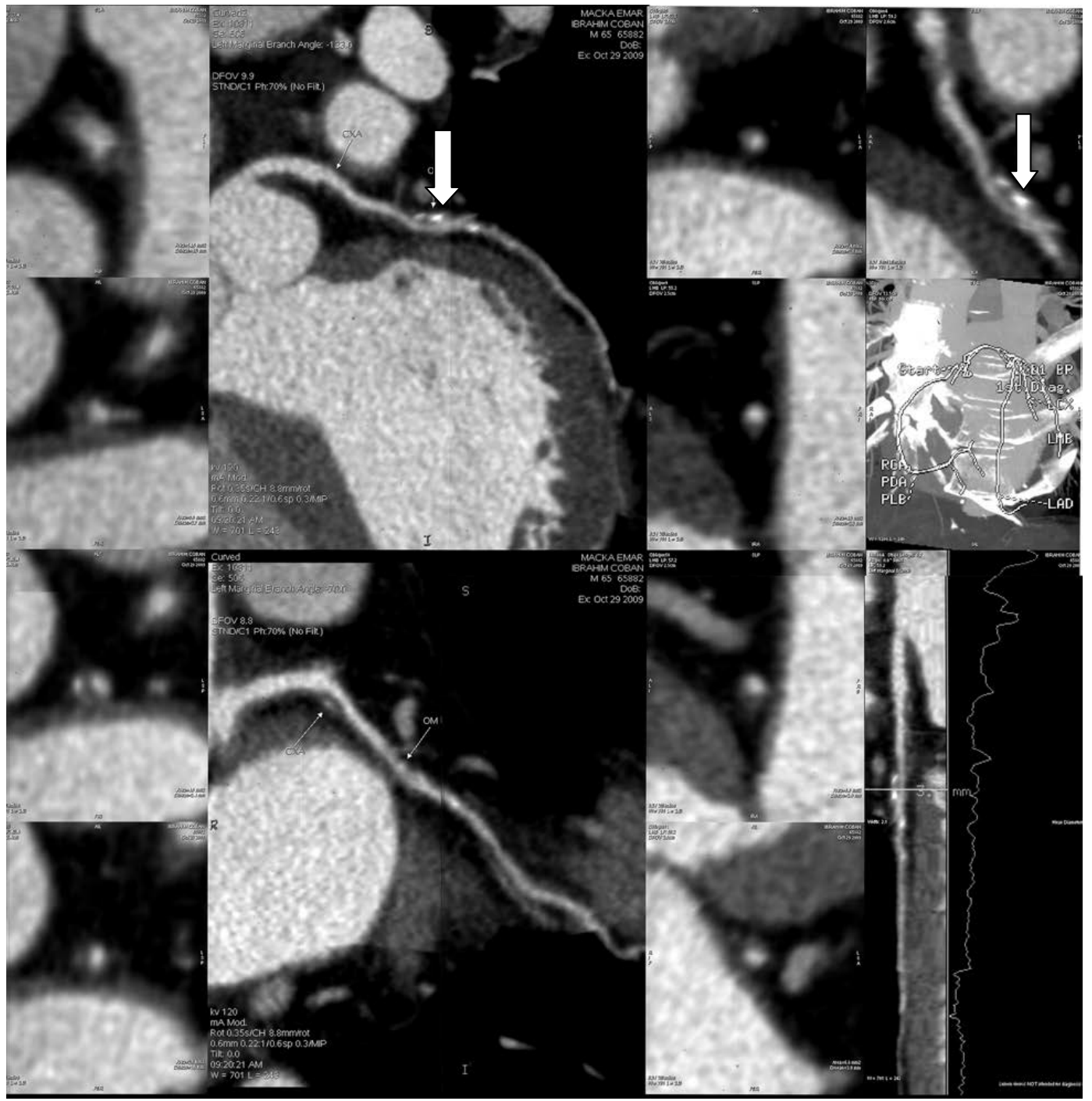

Figure 8: The atheromatous component with small adventitial calcifications surrounded by some degree of lipids (soft plaques) are shown with arrows on the LAD artery.

necessary after CABG surgery or stent implantation in order to prevent the progression of the virulent process. Our combination therapy also makes a major contribution to the treatment of osteoporosis while it prevents diseases such as atherosclerocalsific arteries, calcified cardiac valve diseases, calcified constrictive pericarditis and urinary phosphate calculus. The main purpose of our therapy with Erzengin's Polypill is to prevent the formation of atherosclerosis, Atherosclerosis Obliterans and completely heal the existing disease.

Aggressive anti-lipid and anti-thrombotic treatments are approved (for suitable patients) in the light of recent developments and new knowledge. In addition to these treatments, it is observed that in cases where Menaquinone-7 (Vitamin K2) and Vitamin D3 are taken naturally the arterial calcifications regress, calcium can mobilize from the walls of arteries to the bones. As it is well known, Vitamin $\mathrm{K}$ includes 2 different groups of agents; Phylloquinone (Vitamin K1) and Menaquinone (Vitamin K2). Insufficient ingestion of Vitamin K2 causes atherosclerotic and calcified plaques on the arterial walls and atherosclerotic coronary arteries due to the lack of Matrix Gla-Protein (MGP) carboxylation. That means, sufficient ingestion of Menaquinone-7 (especially Vitamin K2=MK-7) prevents atherosclerotic and calcified plaque formation. Also, according to several researches, it has been observed that the ingestion of Plant Sterol Esters and particularly the esters of Plant Stanol lowers total cholesterol, LDL and triglyceride in serum and cures atherosclerosis in the ratio of 15-27\%. The combination of Plant Stanol Esters and statins prevent atherosclerosis in the ratio of $46 \%$. According to our results; Plant Stanol esters are more effective than the sterols. On the other hand, these esters prevent side effects of statins. The other two components of Erzengin's polypill (Table 1) combinantion are Omega-3 (Eicosapentaenoic Acid - EPA and Docosahexaenoic Acid DHA) and Ubiquinol (Coezym QH).

The beginning of our researches, the compared animal study (Stage A) was initiated at DETAE in Istanbul Medical Faculty.

For the second stage of the researches, we have planned a case study group (Stage B) consisted of patients with informed consent and divided into 2 random subgroups. The first subgroup (Group 


\section{Erzengin's Polypill is:}

$\checkmark \quad$ MENAQUINE-7 (VIT. K 2), $50 \mathrm{mg}$.

$\checkmark \quad$ OMEGA 3 (Eicosapentaenoic Acid-EPA and Docosahexaenoic Acid-DHA), $1200 \mathrm{mg}$.

$\checkmark \quad$ PLANT STANOL ESTERIES, 6 gr.v

$\checkmark \quad$ COENZYM Q10 (Ubiquinol), $100 \mathrm{mg}$. $+$

AGRESSIF ANTILIPIDS,

Table 1: Erzengin's Polypill combination is shown in this table.

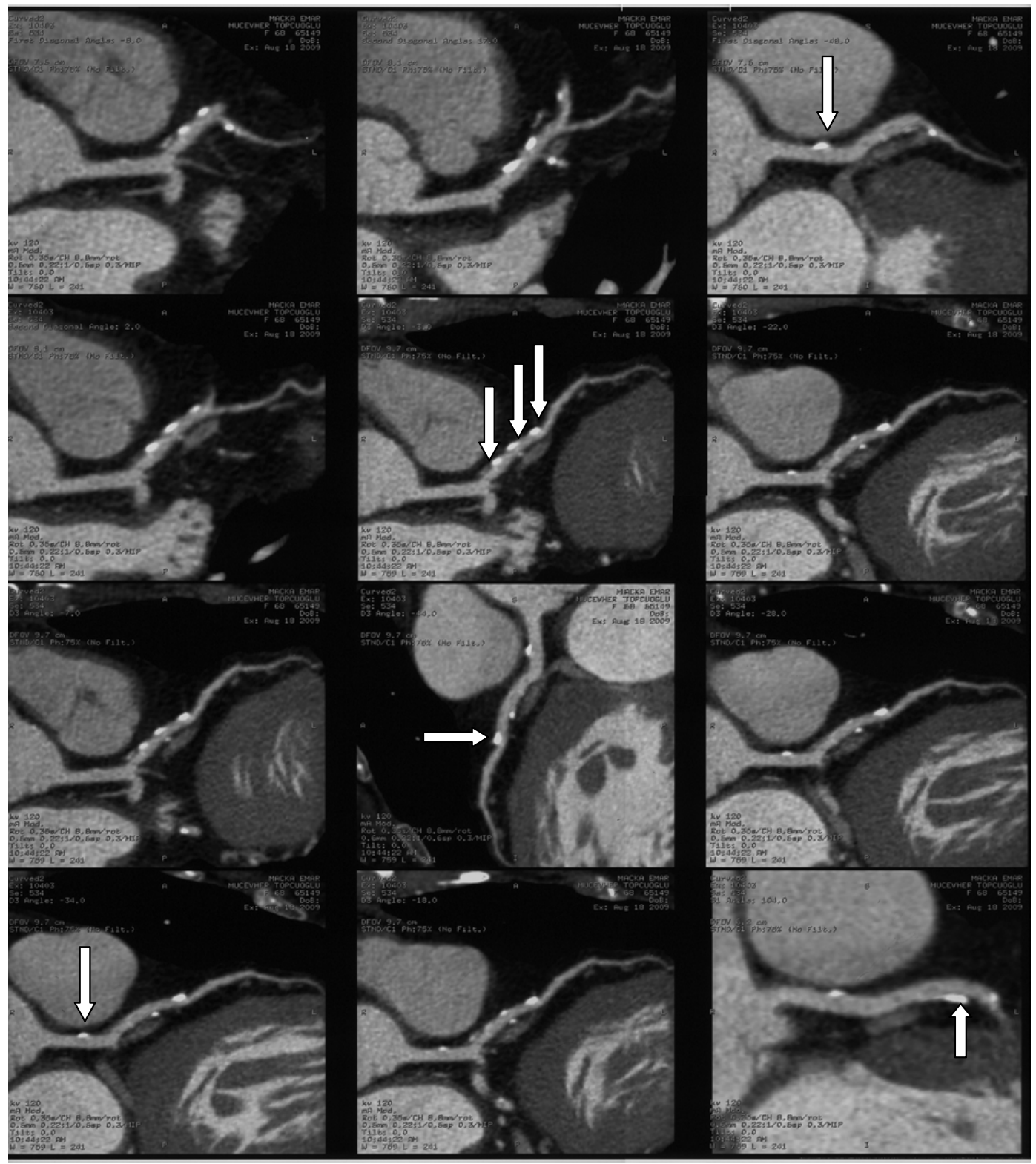

Figure 9: Consecutive many epithelial calcifications looks like beads on the adventitia of the LAD artery and one calcified plaque on the adventitia of Left Main Coronary Artery.

B-1) consists of patients who prefer to have ordinary treatment. These patients are being treated with classical medication in order to make a comparison between the second group of patients. This subgroup (Group B-2) beings medicated with the polypill combination therapy together with the other ordinary therapy.
If the research accomplishes its objectives, there will be a permanent solution for the Atherosclerosis which leads to virulent diseases in arteries of vital organs and sudden deaths. The combination of Erzengin's Polypill (Table 2) together with the ordinary therapy will sort out the complications of the atherosclerosis permanently. We believe that this therapy will completely prevent the atherosclerosis 
*the calcıfıcatıon ınhıbıtor needs to vitamın k2 (menaquınone-7).

In vascular calcıum methabolısm, the menaquınone-7 dependent matrıx gla-proteın (mgp) plays a dominant role.

*It has been shown that mgp is a potent inhıbıtor of arterıal calcificatıon at all three tunıcas of arteries.

*The calcifıcatıon ınhıbıtor needs to vitamın k2 (menaquinone-7).

In vascular calcıum methabolısm, the menaquınone-7 dependent matrıx gla-proteın (mgp) plays a domınant role.

It has been shown that mgp is a potent ınhibitor of arterıal calcıfıcatıon at all three tunıcas of arteries.

*OMEGA-3=

A) Linolenic Acid,

B) Fish Olives:

1)Eicosapentaenoic Acid (EPA)

2)Docosahexaenoic Acid (DHA).

*OMEGA 3 (EPA+DHA):

Is an ımportant agent for treatment of coronary atherosclerosıs. Sınce 1970's the cardıoprotectıf effects of epa \& dha have been well documented *COENZYM QH:

Also it is an Important mediator for necassary energy and, on the other hand to prevent of coronary atherosclerosıs.

*Plant Stanol Esterıes: are also ımportant mediators for prevent of side effect and to reduce daıly dosace of statıns.

Table 2: In this table the mechanism of drugs of Erzengin's Polipill are shown.

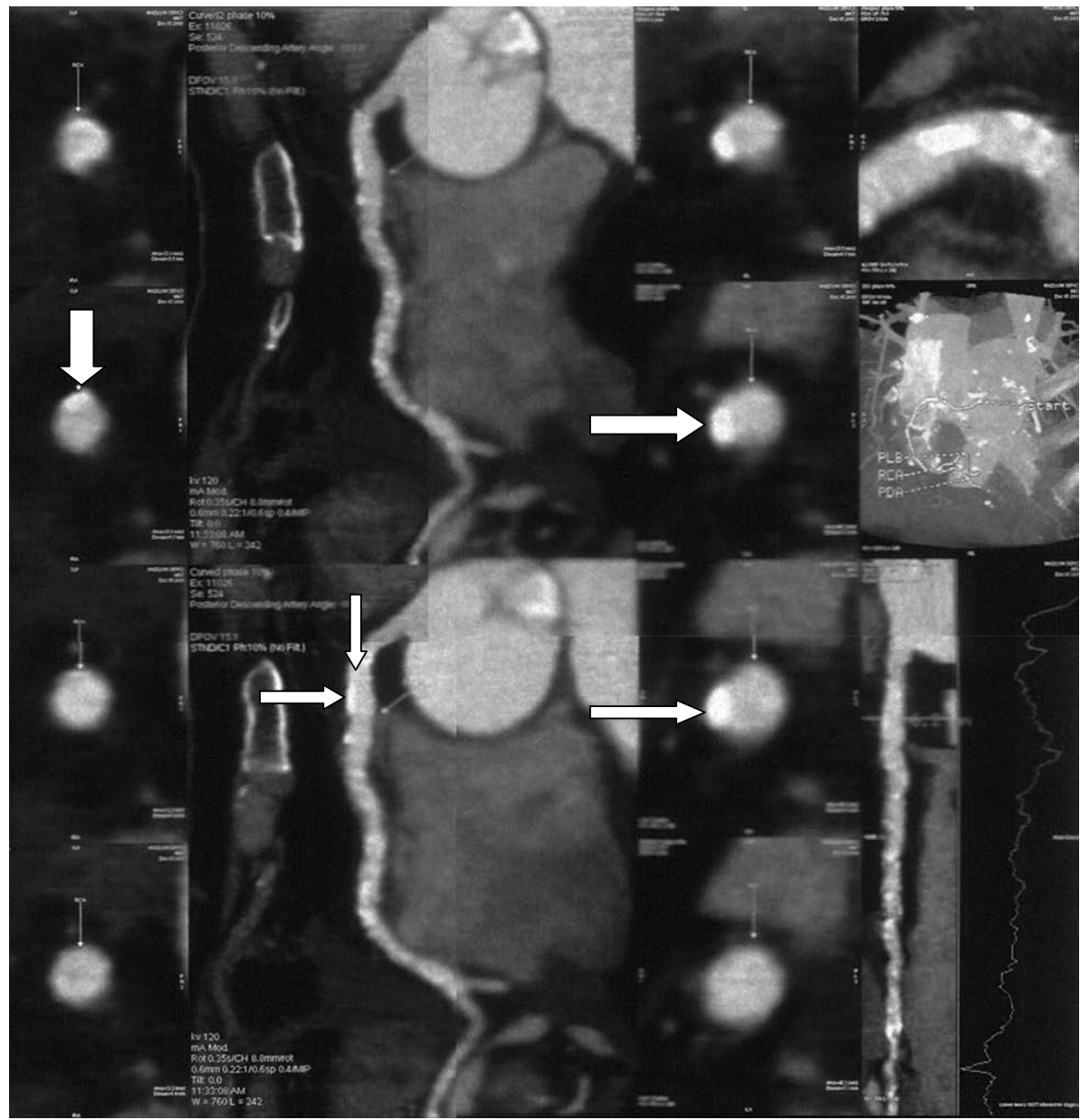

Figure 10: Long and small calcified plaques developed just at the adventitial epithelium on the RCA artery are shown (with arrows) by cross section and long axis views of MSCT.

in a non-invasively, risk free and inexpensive way. Our research will make an incomparable contribution to whole humanity by expanding the life span and enhancing the quality of life as it will provide a remarkable success for science and make a great contribution to the economy.

\section{Conclusion}

Today(in 2013): It is possible to treate coronary (or all arterial) atherosclerocalcifications with only drugs combination therapy by medically.

${ }^{*}$ (An innovative treatment and exact solution of coronaryatherosclero calcifications)

\section{References}

1. Fuster V (1999) Epidemic of cardiovascular disease and stroke: the three main challenges. Presented at the 71st scientific sessions of the American Heart Association. Dallas, Texas. Circulation 99: 1132-1137. 


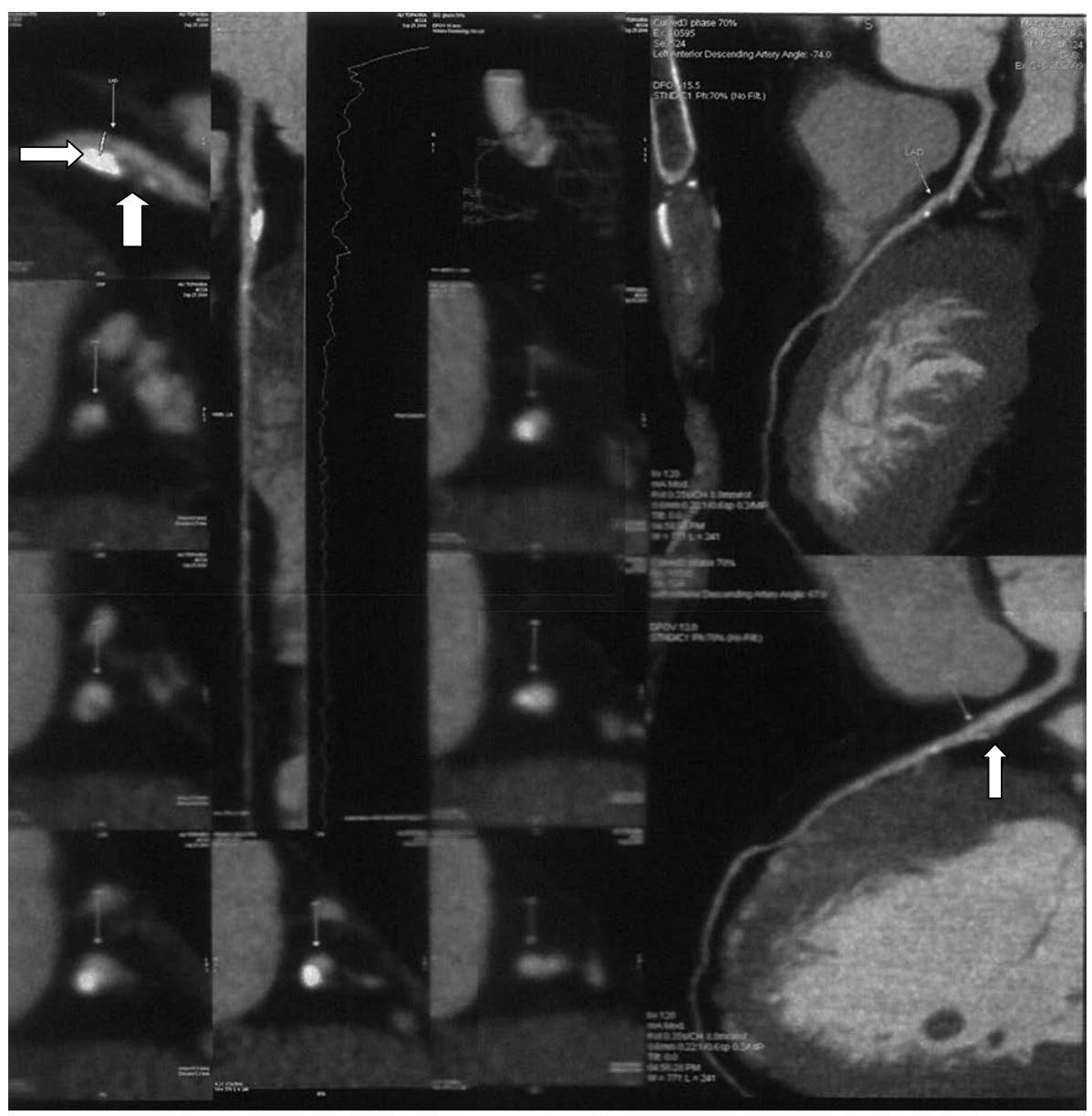

Figure 11: The atheromatous component are shown, with the vertical arrows (lipids core) and medium size adventitial calcifications (the last stop of the atheromatheous process) is shown a horizontal arrow on the LAD artery.

2. Fuster V (2004) Atherosclerosis, thrombosis, and vascular biology. Cecil Textbook of Medicine, Ed: Lee Goldman, Dennis Ausiello, (22 $\left.{ }^{\text {nd }} e d n\right)$, Saunders, Philadelphia 383-400.

3. Prevention of cardiovascular events and death with pravastatin in patient with coronary heart disease and abroad range of initial cholesterol levels. The LongTerm Intervention with Pravastatin in Ischaemic Disease (LIPID) Study Group (1998) N Engl J Med 339: 1349-1357.

4. Falk E, FusterV. Atherogenesis and its determinants. Hurst's The Heart, Ed: Valentin Fuster, R. Wayne Alexander, Robert A. O'Rourke, Robert Roberts.

5. Spencer B King III, Hein JJ Wellens (2001) $\left(10^{\text {th }}\right.$ edn), McGraw-Hill, New York 1: $1065-1093$.

6. Ross R (1998) The biology of atherosclerosis. In: Topol E J, ed. Comprehensive Cardiovascular Medicine. Philadelphia:lippincott-Raven 1998: 13.

7. Leschka S, Alkadhi H, Plass A, Desbiolles L, Grünenfelder J, et al. (2005) Accuracy of MSCT coronary angiography with 64-slice technology: first experience. Eur Heart J 26: 1482-1487.

8. liyama K, Hajra L, liyama M, Li H, Di Chiara M, et al. (1999) Patterns of vascular cell adhesion molecule-1 and intercellular adhesion molecule-1 expression in rabbit and mouse atherosclerotic lesions and at sites predisposed to lesion formation. Circ Res 85: 199-207.

9. Erzengin F, Büyüköztürk K (2007) Cardiac Imaging in Textbook of Internal Medicine, Ed: Kemalettin Büyüköztürk, Faruk Erzengin et all. Nobel, Istanbul 1623-1644.

10. de Ruijter W, Westendorp RG, Assendelft WJ, den Elzen WP, de Craen AJ, et al. (2009) Use of Framingham risk score and new biomarkers to predict cardiovascular mortality in older people: population based observational cohort study. BMJ 338: a3083.

11. (2002) Third Report of the National Cholesterol Educatio Program (NCEP) Expert Panel on Detection, Evaluation and Treatment of High Blood Cholesterol in Adult (Adult Treatment Panel III) final report. Circulation, 106: 3143-421.

12. Conroy RM, Pyörälä K, Fitzgerald AP, Sans S, Menotti A, et al. (2003) Estimation of ten-year risk of fatal cardiovascular disease in Europe: the SCORE project. Eur Heart J 24: 987-1003.

13. Hendel RC, Patel MR, Kramer CM, Poon M, Hendel RC, et al. (2006) ACCF/ ACR/SCCT/SCMR/ASNC/NASCI/SCAI/SIR 2006 appropriateness criteria for cardiac computed tomography and cardiac magnetic resonance imaging: a report of the American College of Cardiology Foundation Quality Strategic Directions Committee Appropriateness Criteria Working Group, American College of Radiology, Society of Cardiovascular Computed Tomography, Society for Cardiovascular Magnetic Resonance, American Society of Nuclear Cardiology, North American Society for Cardiac Imaging, Society for Cardiovascular Angiography and Interventions, and Society of Interventional Radiology. J Am Coll Cardiol 48: 1475-1497.

14. Achenbach S, Daniel WG (2005) Computed Tomography of the Heart Braunwald's Heart Disease,7th edition, Elsevier Saunders, Pensilvania,USA 285-287.

15. Higgins CB, de Roos A (2006) MRI and CT of the Cardiovascular system. Lipincor \&Williams and Wilkins,USA and the Netherlands.

16. Achenbach S, Moselewski F, Ropers D, Ferencik M, Hoffmann U, et al. (2004) Detection of calcified and noncalcified coronary atherosclerotic plaque by contrast-enhanced, submillimeter multidetector spiral computed tomography: a segment-based comparison with intravascular ultrasound. Circulation 109: 14-17. 


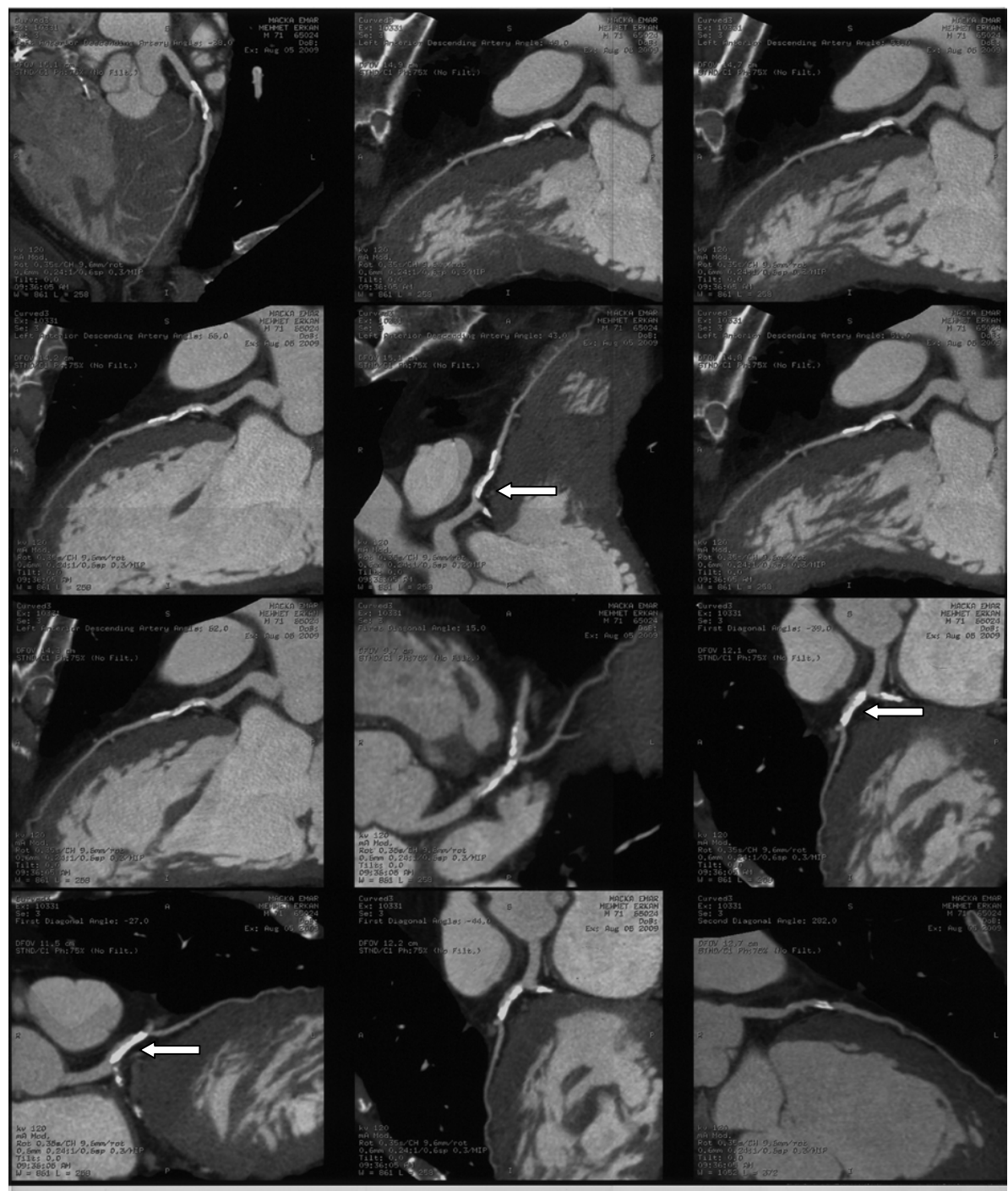

Figure 12: Severe calcification (shown arrows) with significant narrowing of the LAD and Circumflex (Cx) lumens of arteries beginning from adventitia of the vessels.

17. Sangiorgi G, Rumberger JA, Severson A, Edwards WD, Gregoire J, et al. (1998) Arterial calcification and not lumen stenosis is highly correlated with atherosclerotic plaque burden in humans: a histologic study of 723 coronary artery segments using nondecalcifying methodology. J Am Coll Cardiol 31: 126133

18. Mintz GS, Pichard AD, Popma JJ, Kent KM, Satler LF, et al. (1997) Determinants and correlates of target lesion calcium in coronary artery disease: a clinical, angiographic and intravascular ultrasound study. J Am Coll Cardiol 29: 268274.

19. Yönal I, Banzagch M, Özcan M, Adalet K, Erzengin F (2009) Cardiac Computed Tomography; The Unique Noninvasive Method For The Detection Of Coronary Atherosclerotic Plaque Morphology And For The Diagnosis Of Silent Ischemia, Lumen Narrowing Calcification Of Adventitia: With Two Case Reports. The Heart Surgery Forum. A Cardiothoracic Multymedia Journal 12: 69-70.

20. Erzengin F:Adventitial Calcification: Surprise Location. Atherosclerotic and calcified plaques begin and grow up not only beneath the endothelium, but also on the adventitia. 78th European Atherosclerosis Society Congress (78th EAS Congress), June 20-23, 2010, Hamburg, Germany.

21. Erzengin F: Adventitial (Erzengin's) Atherosclrero-calcification: Surprise Location. Atherosclerotic and calcified plaques begin and grow up not only beneath the endothelium, but also on the adventitia. THE 3rd World Medıcıne Congress in abstract book, Page 4 September 20-23, 2010/NALCHIK-RUSSIA.
22. Erzengin F: Adventitial arherosclerocalcifications: A new surprise location In Abstract book, 7th Congress of update in Cardiology and Cardiovascular Surgery in association with TCT Mediterranean 24-27th March, 2011/ANTALYATURKEY.

23. Bissen EA (2011) Plaque Adventitia: Service Hatch or Battleground? 79th EAS Congress Gothenburg, Sweden, June 26-29.

24. Ezengin F: ERZENGIN'S POLYPILL: An additinal medical treatment of the atherosclerocalcifications. The 4th international medical conference on "advances incontemporary medicine" 7-8 October, 2011, ISTANBUL.

25. Erzengin F: Letter to the editor: Adventitial atherosclerosis and calcifications: Cardiovascular Sciences, Accepted for press (07.06.2010- Nm:18915).

26. Erzengin F: Erzengin's Polypill (2012) An additional and new medical treatment of atherosclerocalcifications. International Journal of Cardiology 147: 111-112.

27. lacobellis G, Ribaudo MC, Zappaterreno A, lannucci CV, Leonetti F (2004) Relation between epicardial adipose tissue and left ventricular mass. Am J Cardiol 94: 1084-1087. 